\title{
On Divisibility of Convolutions of Central Binomial Coefficients
}

\author{
Mark R. Sepanski \\ Department of Mathematics, Baylor University \\ One Bear Blace \#97328 \\ Waco, TX 76798-7328, U.S.A. \\ Mark_Sepanski@baylor.edu
}

Submitted: May 5, 2013; Accepted: Jan 11, 2014; Published: Feb 13, 2014

Mathematics Subject Classifications: 11B65, 05A10

\begin{abstract}
Recently, Z. Sun proved that

$$
2(2 m+1)\left(\begin{array}{c}
2 m \\
m
\end{array}\right) \mid\left(\begin{array}{c}
6 m \\
3 m
\end{array}\right)\left(\begin{array}{c}
3 m \\
m
\end{array}\right)
$$
\end{abstract}

for $m \in \mathbb{Z}_{>0}$. In this paper, we consider a generalization of this result by defining

$$
b_{n, k}=\frac{2^{k}(n+2 k-2) ! !}{(n-2) ! ! k !} .
$$

In this notation, Sun's result may be expressed as $2(2 m+1) \mid b_{(2 m+1),(2 m+1)-1}$ for $m \in \mathbb{Z}_{>0}$. In this paper, we prove that

$$
2 n \mid b_{n, u n \pm 2^{r}}
$$

for $n \in \mathbb{Z}_{>0}$ and $u, r \in \mathbb{Z}_{\geqslant 0}$ with $u n \pm 2^{r}>0$. In addition, we prove a type of converse. Namely, fix $k \in \mathbb{Z}$ and $u \in \mathbb{Z}_{\geqslant 0}$ with $u>0$ if $k<0$. If

$$
2 n \mid b_{n, u n+k}
$$

for all $n \in \mathbb{Z}_{>0}$ with $u n+k>0$, then there exists a unique $r \in \mathbb{Z}_{\geqslant 0}$ so that either

$$
k=2^{r} \text { or } k=-2^{r} .
$$

Keywords: central binomial coefficients 


\section{Introduction}

There has been much recent work on topics relating to central binomial coefficients $([1,2,3,5,6,7,8,9,10,11,12])$. In particular, Z. Sun in [9] proved interesting results on congruences of sums of products of central binomial coefficients. One such result is that $2(2 m+1)\left(\begin{array}{c}2 m \\ m\end{array}\right) \mid\left(\begin{array}{c}6 m \\ 3 m\end{array}\right)\left(\begin{array}{c}3 m \\ m\end{array}\right)$ for $m \in \mathbb{Z}_{>0}$. The new integer sequence $m \rightarrow$ $\left(\begin{array}{c}6 m \\ 3 m\end{array}\right)\left(\begin{array}{c}3 m \\ m\end{array}\right) /\left[2(2 m+1)\left(\begin{array}{c}2 m \\ m\end{array}\right)\right]$ is given by A176898 in the OIES. Using this sequence, Sun proposed a number of open conjectures ([9, Conjectures 2, 4, 6 and 8]) on certain divisibility properties of this and related sequences.

In this paper, we consider a generalization of Sun's new sequence. Recognizing $\left(\begin{array}{c}6 m \\ 3 m\end{array}\right)\left(\begin{array}{c}3 m \\ m\end{array}\right) /\left(\begin{array}{c}2 m \\ m\end{array}\right)$ as the coefficient of $x^{2 m}$ in the $(2 m+1)$-fold convolution of the central binomial sequence with itself, we define $b_{n, k}$ to be the $k^{\text {th }}$ term of the $n$-fold convolution of the central binomial sequence with itself--which turns out to be

$$
b_{n, k}=\frac{2^{k}(n+2 k-2) ! !}{(n-2) ! ! k !} .
$$

In this notation, Sun's result is that $2(2 m+1) \mid b_{2 m+1,(2 m+1)-1}$ for $m \in \mathbb{Z}_{>0}$.

In this paper (Theorem 1), we prove that

$$
2 n \mid b_{n, u n \pm 2^{r}}
$$

for $n \in \mathbb{Z}_{>0}$ and $u, r \in \mathbb{Z}_{\geqslant 0}$ with un $\pm 2^{r}>0$. In particular, Sun's result is a special case of the above theorem in which $n=2 m+1, u=1, r=0$, and the - sign is chosen. In addition, we prove a type of converse (Theorem 2). Namely, fix $k \in \mathbb{Z}$ and $u \in \mathbb{Z} \geqslant 0$ with $u>0$ if $k<0$. If

$$
2 n \mid b_{n, u n+k}
$$

for all $n \in \mathbb{Z}_{>0}$ with $u n+k>0$, then there exists a unique $r \in \mathbb{Z}_{\geqslant 0}$ so that either

$$
k=2^{r} \text { or } k=-2^{r} .
$$

\section{Definition}

Write $b$ for the sequence of central binomial coefficients, $b_{j}=\left(\begin{array}{c}2 j \\ j\end{array}\right)$, with $j \in \mathbb{Z}_{\geqslant 0}$. For $n, k \in \mathbb{Z}_{\geqslant 0}$ with $n \geqslant 1$, we define the doubly indexed sequence $b_{n, k} \in \mathbb{Z}_{>0}$ to be the $k^{\text {th }}$ term of the $n$-fold convolution of $b$ with itself, $b_{n, k}=\left(b^{* n}\right)_{k}$. In the degenerate case of $n=0$, we define $b_{0,0}=1$ and $b_{0, k}=0$ for $k>0$. It follows that the generating function for the sequence $k \rightarrow b_{n, k}$ is $(1-4 x)^{-n / 2}$ and a trivial calculation shows that

$$
b_{n, k}=\frac{2^{k}(n+2 k-2) ! !}{(n-2) ! ! k !}
$$

when $n \geqslant 2$. For use in Theorem 2 and in order to compare with [9], we note that it is straightforward to verify that

$$
b_{2 m, k}=2^{2 k}\left(\begin{array}{c}
m+k-1 \\
k
\end{array}\right), \quad b_{2 m+1, k}=\frac{\left(\begin{array}{c}
2 m+2 k \\
m+k
\end{array}\right)\left(\begin{array}{c}
m+k \\
m
\end{array}\right)}{\left(\begin{array}{c}
2 m \\
m
\end{array}\right)}
$$


for $m \in \mathbb{Z}_{>0}$ in the first formula above and $m \in \mathbb{Z}_{\geqslant 0}$ in the second.

\section{Divisibility}

Here we present the main result on the divisibility of the sequence $b_{n, k}$.

Theorem 1. For $n \in \mathbb{Z}_{>0}$ and $u, r \in \mathbb{Z}_{\geqslant 0}$,

$$
2 n \mid b_{n, u n+2^{r}}
$$

and, if also un $-2^{r}>0$,

$$
2 n \mid b_{n, u n-2^{r}} .
$$

Proof. Begin with the convolution definition

$$
b_{n, u n \pm 2^{r}}=\sum_{\substack{a_{1}, \ldots, a_{n} \in \mathbb{Z}_{\geqslant 0} \\
a_{1}+\cdots+a_{n}=u n \pm 2^{r}}} \prod_{j=1}^{n}\left(\begin{array}{c}
2 a_{j} \\
a_{j}
\end{array}\right)
$$

and let $X=\left\{\left(a_{1}, \ldots, a_{n}\right) \in \mathbb{Z}_{\geqslant 0}^{n} \mid a_{1}+\cdots+a_{n}=u n \pm 2^{r}\right\}$. Observe that $0 \notin X$ since un $+2^{r}$, un $-2^{r} \geqslant 1$.

The set $X$ carries a natural action of the symmetric group, $S_{n}$, acting by permuting the coordinates. Write $\mathcal{O}_{1}, \ldots, \mathcal{O}_{N}$ for the orbits of $X$ under the action of $S_{n}$. Clearly each $\mathcal{O}_{k}, 1 \leqslant k \leqslant N$, has a unique representative of the form

$$
x_{k}=(\overbrace{c_{1 k}, \ldots c_{1 k}}^{d_{1 k}}, \overbrace{c_{2 k}, \ldots, c_{2 k}}^{d_{2 k}}, \ldots, \overbrace{c_{m_{k} k}, \ldots c_{m_{k} k}}^{d_{m_{k} k}})
$$

with $0 \leqslant c_{1 k}<c_{2 k}<\cdots<c_{m_{k} k}$ and $d_{j k}, m_{k} \in \mathbb{Z}_{>0}$. Since the stabilizer of $x_{k}$ in $S_{n}$ is clearly isomorphic to $S_{d_{1 k}} \times S_{d_{2 k}} \times \cdots \times S_{d_{m_{k} k}}$, if follows that

$$
\left|\mathcal{O}_{k}\right|=\frac{n !}{d_{1 k} ! d_{2 k} ! \cdots d_{m_{k} k} !}=\left(\begin{array}{c}
n \\
d_{k}
\end{array}\right)
$$

where we use multinomial notation above and write $d_{k}=\left(d_{1 k}, d_{2 k}, \ldots, d_{m_{k} k}\right)$. Using this, we may rewrite the formula for $b_{n, u n \pm 2^{r}}$ as

$$
b_{n, u n \pm 2^{r}}=\sum_{k=1}^{N}\left(\begin{array}{c}
n \\
d_{k}
\end{array}\right) \prod_{j=1}^{m_{k}}\left(\begin{array}{c}
2 c_{j k} \\
c_{j k}
\end{array}\right)^{d_{j k}} .
$$

We will prove the theorem by demonstrating that $2 n \mid\left(\begin{array}{c}n \\ d_{k}\end{array}\right) \prod_{j=1}^{m_{k}}\left(\begin{array}{c}2 c_{j k} \\ c_{j k}\end{array}\right)^{d_{j k}}$ for each $k$. 
As $x_{k} \in X$, it follows that

$$
\begin{gathered}
\sum_{j=1}^{m_{k}} d_{j k}=n \\
\sum_{j=1}^{m_{k}} d_{j k} c_{j k}=u n \pm 2^{r} .
\end{gathered}
$$

Therefore,

$$
\sum_{j=1}^{m_{k}} d_{j k}\left(c_{j k}-u\right)= \pm 2^{r}
$$

and we may write the greatest common divisor of $d_{1 k}, d_{2 k}, \ldots, d_{m_{k} k}$ as $2^{q_{k}}$ for some $q_{k}$, $0 \leqslant q_{k} \leqslant r$. Since it follows that $2^{q_{k}} \mid n$, we also see that $2^{q_{k}} \leqslant n$.

Choose $w_{j k} \in \mathbb{Z}, 1 \leqslant j \leqslant m_{k}$, so that $\sum_{j=1}^{m_{k}} w_{j k} d_{j k}=2^{q_{k}}$. Write $e_{j}$ for the $j^{\text {th }}$ standard basis vector, $e_{j}=(\overbrace{0, \ldots 0,1}^{j}, 0, \ldots 0) \in \mathbb{Z}^{m_{k}}$ (suppressing the $m_{k}$ dependence). Then

$$
\begin{aligned}
2^{q_{k}}\left(\begin{array}{c}
n \\
d_{k}
\end{array}\right) & =\sum_{j=1}^{m_{k}} w_{j k} d_{j k}\left(\begin{array}{c}
n \\
d_{k}
\end{array}\right)=\sum_{\substack{j=1 \\
d_{j k} \geqslant 1}}^{m_{k}} w_{j k} d_{j k}\left(\begin{array}{c}
n \\
d_{k}
\end{array}\right) \\
& =\sum_{\substack{j=1 \\
d_{j k} \geqslant 1}}^{m_{k}} w_{j k}\left(\begin{array}{c}
n-1 \\
d_{k}-e_{j}
\end{array}\right) n
\end{aligned}
$$

so that $n \mid 2^{q_{k}}\left(\begin{array}{c}n \\ d_{k}\end{array}\right)$ and

$$
2 n \mid\left(\begin{array}{c}
n \\
d_{k}
\end{array}\right) 2^{q_{k}+1} .
$$

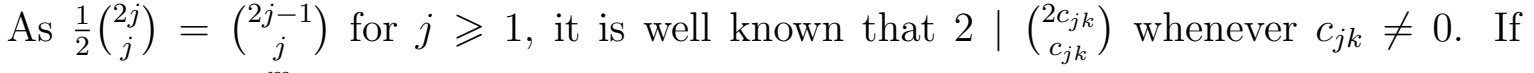
$c_{1 k} \neq 0$, then $2^{n} \mid \prod_{j=1}^{m_{k}}\left(\begin{array}{c}2 c_{j k} \\ c_{j k}\end{array}\right)^{d_{j k}}$. As $2^{q_{k}+1} \leqslant 2 n \leqslant 2^{n}$, it follows that $2 n \mid\left(\begin{array}{c}n \\ d_{k}\end{array}\right) \prod_{j=1}^{m_{k}}\left(\begin{array}{c}2 c_{j k} \\ c_{j k}\end{array}\right)^{d_{j k}}$ and we are done.

Suppose, therefore, that we are in the case of $c_{1 k}=0$ (so $m_{k} \geqslant 2$ ). Let $s_{k}=n-d_{1 k}$. Now all that we get is that $2^{s_{k}} \mid \prod_{j=1}^{m_{k}}\left(\begin{array}{c}2 c_{j k} \\ c_{j k}\end{array}\right)^{d_{j k}}$. If $q_{k}+1 \leqslant s_{k}$, then a similar argument as in the above paragraph shows $2 n \mid\left(\begin{array}{c}n \\ d_{k}\end{array}\right) \prod_{j=1}^{m_{k}}\left(\begin{array}{c}2 c_{j k} \\ c_{j k}\end{array}\right)^{d_{j k}}$ and we are done. It remains only to show that $q_{k}+1 \leqslant s_{k}$. So suppose that $q_{k}+1>s_{k}$ and write $d_{j k}=2^{q_{k}} t_{j k}$ for $t_{j k} \in \mathbb{Z}_{>0}$. Since we must have $t_{j k} \geqslant j-1 \geqslant 1$ when $j \geqslant 2$, we get

$$
q_{k}+1>s_{k}=\sum_{j=2}^{m_{k}} d_{j k}=\sum_{j=2}^{m_{k}} 2^{q_{k}} t_{j k} \geqslant \sum_{j=2}^{m_{k}} 2^{q_{k}}=2^{q_{k}}\left(m_{k}-1\right) \geqslant 2^{q_{k}} .
$$

Since it is impossible to obtain $q+1>2^{q}$, we arrive at the desired contradiction. 
For the case of $2 n \mid b_{n, u n+2^{r}}$ in the above theorem, $n=0$ is ruled out in order to make sure that division by $2 n$ is well defined (note $b_{0,0}=1$ and $b_{0, k}=0$ for $k>0$ ). For the case of $2 n \mid b_{n, u n-2^{r}}$, we require $u n>2^{r}$ since $b_{n, 0}=1$.

We also note that Sun's result

$$
2(2 m+1)\left(\begin{array}{c}
2 m \\
m
\end{array}\right) \mid\left(\begin{array}{c}
6 m \\
3 m
\end{array}\right)\left(\begin{array}{c}
3 m \\
m
\end{array}\right)
$$

for $m \in \mathbb{Z}_{>0}$ is equivalent to

$$
2(2 m+1) \mid \frac{\left(\begin{array}{c}
6 m \\
3 m
\end{array}\right)\left(\begin{array}{c}
3 m \\
m
\end{array}\right)}{\left(\begin{array}{c}
2 m \\
m
\end{array}\right)} .
$$

This is then a special case of our equation $2 n \mid b_{n, u n-2^{r}}$ in which $n=2 m+1, u=1$, and $r=0$. This gives the same statement as the above equation, but written as

$$
2(2 m+1) \mid b_{2 m+1,2 m} .
$$

\section{A Type of Converse}

The next result is a type of converse to Theorem 1.

Theorem 2. Fix $k \in \mathbb{Z}$ and $u \in \mathbb{Z}_{\geqslant 0}$ with $u>0$ if $k<0$. If

$$
2 n \mid b_{n, u n+k}
$$

for all $n \in \mathbb{Z}_{>0}$ with un $+k>0$, then there exists a unique $r \in \mathbb{Z}_{\geqslant 0}$ so that either

$$
k=2^{r} \text { or } k=-2^{r} .
$$

Proof. First we show that $k \neq 0$. For this choose any odd prime $p$ and consider $b_{2 p, 2 u p}$. Using Theorem 4 of [4] and the Division Algorithm, work $\bmod p$ to see that

$$
b_{2 p, 2 u p}=2^{4 u p}\left(\begin{array}{c}
(1+2 u) p-1 \\
p-1
\end{array}\right) \equiv 2^{4 u}\left(\begin{array}{c}
2 u \\
0
\end{array}\right)\left(\begin{array}{c}
p-1 \\
p-1
\end{array}\right) \equiv 2^{4 u} .
$$

Thus $p \nmid b_{2 p, 2 u p}$ and so $k \neq 0$.

Next we consider the case of $u=0$ (so $k>0$ here). Actually the following argument works whenever $k>0$ so that is all we actually assume. If $k$ has an odd prime divisor, $p$, write $k=p k^{\prime}$ for some $k^{\prime} \in \mathbb{Z}_{>0}$. Consider $b_{2 p, 2 u p+k}$. Then, working $\bmod p$ again,

$$
b_{2 p, 2 u p+k}=2^{2\left(2 u+k^{\prime}\right) p}\left(\begin{array}{c}
\left(1+2 u+k^{\prime}\right) p-1 \\
p-1
\end{array}\right) \equiv 2^{2\left(2 u+k^{\prime}\right)}\left(\begin{array}{c}
2 u+k^{\prime} \\
0
\end{array}\right)\left(\begin{array}{c}
p-1 \\
p-1
\end{array}\right) \equiv 2^{2\left(2 u+k^{\prime}\right)} .
$$

Thus $p \nmid b_{2 p, 2 u p+k}$ and so $k$ must be a power of 2 . Note that the only reason this argument may fail for $k<0$ is that we might have $2 u p+k \leqslant 0$.

Finally, consider the case of $u \neq 0$. Suppose there exists an odd prime $p$ so $p \mid k$. Then write $k=p k^{\prime}$ and fix $m_{0} \in \mathbb{Z}_{\geqslant 0}$ so $2^{m_{0}+1} u+k^{\prime}>0$ and $2^{m_{0}+1} u$ is congruent $\bmod p$ 
to either 0 or 1 (depending on whether $p \mid u$ or $p \nmid u$ ). Now consider $n=2^{m_{0}+1} p\left(p^{N}+1\right)$ for any sufficiently large $N$. We will show that $p \nmid b_{n, u n+k}$. For this, write

$$
\begin{aligned}
u n+k & =2^{m_{0}+1} u p\left(p^{N}+1\right)+k^{\prime} p \\
& =2^{m_{0}+1} u p^{N+1}+\left(2^{m_{0}+1} u+k^{\prime}\right) p .
\end{aligned}
$$

For sufficiently large $N$, un $+k$ can be expanded in base $p$ as

$$
\begin{aligned}
u n+k & =a_{r} p^{r}+a_{r-1} p^{r}+\cdots+a_{N+1} p^{N+1} \\
& +b_{s} p^{s}+b_{s-1} p^{s-1}+\cdots+b_{1} p
\end{aligned}
$$

with $0 \leqslant a_{i}, b_{j} \leqslant p-1, a_{N+1} \leqslant 1$, and $s \leqslant N$. Now we apply Kummer's theorem to the binomial coefficient in

$$
b_{n, u n+k}=2^{p\left(p^{N}+1\right)}\left(\begin{array}{c}
p^{N+1}+p-1+u n+k \\
p^{N+1}+p-1
\end{array}\right) .
$$

Clearly adding $u n+k$ to $p^{N+1}+(p-1)$ in base $p$ results in no carries so that $p \nmid b_{n, u n+k}$. As a result, $k$ has no odd prime divisors and we are done.

\section{Relation to Known Sequences}

As a result of Theorem 1, we have the following integer sequences

$$
n \rightarrow B_{n, u, r, \pm} \equiv \frac{b_{n, u n \pm 2^{r}}}{2 n}
$$

For most choices of parameters $u, r, \pm$, this sequence seems to be new. However, for a few special choices, the sequence is known. Up to a shift and a few initial terms, the sequence $B_{n, 0,2,+}$ is the OEIS integer sequence A077415, $B_{n, 1,0,+}$ is A085614, $B_{n, 1,1,+}$ is A078531, and $B_{n, 1,0,-}$ appears as every other term in A089073. In addition, the odd terms of $B_{n, 0,2,+}$ are A162540, the even terms of $B_{n, 0,2,+}$ are A102860 and the negative of A136264, and (by construction) the odd terms of $B_{n, 1,0,-}$ are Sun's A176898.

\section{$6 \quad$ Final Remarks}

It would be interesting to find a combinatorial interpretation for the sequences $B_{n, u, r, \pm}$. For instance, one is given the case of $B_{n, 1,0,-}(\mathrm{A} 089073)$ or $B_{n, 1,1,+}$ (A078531) as the number of symmetric non-crossing connected graphs on equidistant nodes of a circle and $B_{n, 1,0,+}(\mathrm{A} 085614)$ is the number of elementary arches of size $n$.

In addition, information on corresponding generating functions would be of interest. Some are known. For example $B_{n, 1,0,+}(\mathrm{A} 085614)$ is the series reversion of $x-3 x^{2}+2 x^{3}$. 


\section{References}

[1] Boyadzhiev, K., Series with central binomial coefficients, Catalan numbers, and harmonic numbers, J. Integer Seq. 15, no. 1, Article 12.1.7, 11 pp., 2012.

[2] Chu, W., and Zheng, D., Infinite series with harmonic numbers and central binomial coefficients, Int. J. Number Theory 5, no. 3, 429-448, 2009.

[3] Ferrari, L., Some combinatorics related to central binomial coefficients: Grand-Dyck paths, coloured noncrossing partitions and signed pattern avoiding permutations, Graphs Combin. 26, no. 1, 51-70, 2010.

[4] Fuchs, D., and Fuchs, M., The arithmetic of binomial coefficients [Kvant 1970, no. 6, 17-25] in Kvant selecta: algebra and analysis, I, 1-12, Math. World, 14, Amer. Math. Soc., Providence, RI, 1999.

[5] Garcia Armas, M., and Sethuraman, B., A note on the Hankel transform of the central binomial coefficients, J. Integer Seq. 11, no. 5, Article 08.5.8, 9 pp, 2008.

[6] Sprugnoli, R., Sums of reciprocals of the central binomial coefficients, Integers 6, A27, 18 pp, 2006.

[7] Straub, A., Moll, V., and Amdeberhan, T., The p-adic valuation of k-central binomial coefficients, Acta Arith. 140, no. 1, 31-42, 2009.

[8] Sun, Z., On congruences related to central binomial coefficients, J. Number Theory 131, no. 11, 2219-2238, 2011.

[9] Sun, Z., Products and sums divisible by central binomial coefficients, Electron. J. Combin. 20(1), P9, 2013.

[10] Sun, Z., Supercongruences involving products of two binomial coefficients, Finite Fields Appl. 22, 24-44, 2013.

[11] Sun, Z., and Tauraso, R., New congruences for central binomial coefficients, Adv. in Appl. Math. 45, no. 1, 125-148, 2010.

[12] Tauraso, R., More congruences for central binomial coefficients, J. Number Theory 130, no. 12, 2639-2649, 2010. 